\title{
OPINIÓN PÚBLICA Y REPRESENTACIONES SOCIALES EN TORNO A LOS ALIMENTOS TRANSGÉNICOS*
}

\author{
ELENA ESPEITX BERNAT y JUAN JOSÉ CÁCERES NEVOT \\ Universidad de Barcelona
}

\author{
PALABRAS CLAVE ADICIONALES \\ ADDITIONAL KEYWORDS \\ Biotecnología, Percepción social, Sociedad, Biotechnology, Social Perception, Society, \\ Alimentación. \\ Food.
}

RESUMEN. La opinión pública tiene una influencia relevante en las decisiones institucionales. En el caso de los organismos genéticamente modificados (OGM), los posicionamientos mayoritariamente opuestos que reflejan las encuestas de opinión desarrolladas en Europa han sido clave en el freno a la autorización de nuevas variedades. Sin embargo, no está claro que esas opiniones se sustenten en posicionamientos manifiestamente adversos de los ciudadanos, sino que operan en un universo social y cultural complejo, en el que la interpretación del debate se enmarca en la percepción que se tiene de la propia sociedad. Por ello conviene analizar qué representaciones sociales los OGM movilizan, para buscar las pautas que sustentan ese posicionamiento y comprobar hasta qué punto la interpretación de la opinión publica se hace verdadero eco.

ABSTRACT. The public opinion has an outstanding influence in the political decisions. The European surveys indicates that the attitudes about OGMs are mainly opposed and these results have been one of the keys in the brake to the authorizations of new varieties. Nevertheless, it is doubtful that those opinions are sustained in adverse stances of the citizens. They operate in a social universe where the interpretation of the discussion is closely bound to the social perceptions about the own society. Therefore, it agrees to approach what kind of social representations mobilize the OMGs, in order to look for the guidelines that sustain the attitudes and to verify if the interpretation of the public opinion publishes recover them.

- El presente artículo se basa en los resultados obtenidos en el proyecto financiado por el Ministerio de Ciencia y Tecnología, El impacto de la biotecnologia en España: la percepción social de las aplicaciones alimentarias. BIO2000-0947. No es el objetivo desarrollar una descripción de los resultados obtenidos, sino abordar las relaciones entre opinión pública y representaciones sociales basándonos en los resultados del mismo. Es, por lo tanto, el artículo el producto de una reelaboración de sus resultados atendiendo al tema analizado.

E-mail: contacte@observatori.info

Revista Internacional de Sociología (RIS)

Tercera Época, No40, Enero-Abril, 2005, pp. 207-228. 
RIS

REVISTA INTERNACIONAL DE SOCIOLOCIA

No 40, ENERO-ABRIL, 2005

ELENA ESPEITX Y JUAN JOSÉ CÁCERES

\section{INTRODUCCIÓN}

Si se define convencionalmente la opinión pública como una síntesis de las opiniones de una determinada sociedad alrededor de un tema concreto, cabe preguntarse cuál es el grado de relación entre aquello que se dice que la población piensa del tema y las representaciones sociales que se detectan alrededor del mismo, sobre todo porque el peso de la opinión pública incide fuertemente en las decisiones institucionales. Un ejemplo elocuente en este sentido lo proporcionan los Organismos Genéticamente Modificados (OGM), frente a los cuales la opinión pública de la Unión Europea (UE) expresó y sigue expresando un elevado rechazo, motivando en su día la suspensión temporal de la autorización de la producción de nuevas variedades, en medio de grandes polémicas entre los agentes favorables y los contrarios a esta nueva tecnología; polémicas, por otro lado, cuyo objetivo fundamental erá orientar la posición de esa opinión pública hacia los postulados defendidos por los distintos agentes para intentar obtener el marco legal más cercano a sus intereses respectivos. Además, en el transcurso de tales debates se han hecho a menudo interpretaciones sobre lo que los consumidores (ciudadanos) piensan, creen e imaginan, a fin de explicar el posicionamiento revelado como mayoritario. Desde esta perspectiva, parece que se considera que la opinión pública y las representaciones sociales tienen una relación estrecha, siendo casi equivalentes. Pero no es así.

Aunque es evidente que en las representaciones sociales reposan las actitudes individuales, la opinión pública no es una mera expresión de esas actitudes, sino algo diferente. La opinión pública se construye mediante una consulta estructurada de posicionamientos que siguen un patrón predetermidado, construido por instituciones o grupos de interés. Para ello se hace uso de encuestas de opinión (sobre todo, los eurobarómetros en el caso que nos ocupa), que incorporan determinados marcadores de posición a fin de dar cuerpo al mensaje emitido desde la sociedad. Los resultados de estas encuestas han servido a menudo para hacer grandes caracterizaciones sociales, entre las cuales sobresale la de una Europa reticente y escéptica ante las promesas de la biotecnología, frente a unos Estados Unidos favorables y tecnoentusiastas.

Por el contrario, las representaciones sociales no se estructuran de manera dirigida, sino que fluyen espontáneamente y sobre ellas operan numerosas influencias. Por ello, es necesario disponer de mejores análisis para aventurarse tanto, pues ni la posición de la sociedad frente a las biotecnologías es una cuestión tan simple como para reducirla a posiciones de "a favor" o "en contra" - ni en Europa, ni en América-, ni se debe confundir lo que son instrumentos de interpretación de la opinión pública con estudios de análisis social. Es justamente esta última confusión la que frecuentemente ha impedido disponer de análisis más profundos sobre la cuestión, pues las encuestas de opinión son con demasiada frecuencia la base exclusiva en los que se apoyan. Tampoco ha sido suficiente contextua- 
lizar los indicadores recogidos o las actitudes de agentes concretos mediante el recurso a ciertas elaboraciones que forman parte del bagaje reciente de disciplinas dedicadas al estudio de las relaciones entre ciencia y sociedad o de la percepción social del riesgo ${ }^{1}$. Y lo que es peor, buena parte de los análisis realizados sobre este tema se han situado claramente en un papel de mediadores, para ver cómo se hace digerible a la opinión pública una posición favorable o desfavorable a los OGM. Flaco favor social éste que, sumado al contexto, contribuye a la situación de irresoluble estancamiento que vive hoy el debate social sobre los $\mathrm{OGM}^{2}$.

Con la intención de ampliar las consideraciones hechas hasta aquí, proponemos desarrollar un análisis de las representaciones sociales de los OGM, considerando, por un lado, la lógica del debate que las induce y, por otro, el conjunto de representaciones donde se inscriben. Más allá de la posición final, nos interesa conocer cómo ésta se construye y cómo se modifica; para ello hay que lograr salir fuera de los marcos encorsetados en que a veces se fija el análisis social de los OGM y abrirlo a la exploración de los ejes de las representaciones sociales.

\section{LA POLÉMICA DE LOS OGMS EN LOS MEDIOS DE COMUNICACIÓN}

\section{Surgimiento y evolución}

En primer lugar, hay que presentar cuál ha sido la evolución del tratamiento mediático de los OGM a lo largo de los últimos años. Para ello, la mejor fuente es la prensa escrita, al haber sido uno de los ámbitos que más interés han despertado para abordar el impacto de los medios de comunicación en la formación de actitudes sociales, muy especialmente a raíz de diferentes situaciones de crisis sanitarias (legionella, ataques bioterroristas con anthrax...), crisis alimentarias (vacas locas, aceite de orujo...), descubrimientos científicos (mapa del genoma humano, investigación con células madre...), avances tecnológicos (clonación humana...), etc. Y lo mismo ha sucedido con los alimentos transgénicos, cuya presencia en los medios de comunicación ha suscitado a menudo reclamaciones desde algunos ámbitos - particularmente, de los ámbitos favorables a su desarrollo— para que

\footnotetext{
${ }^{1}$ Por citar un ejemplo interesante, ver López y Luján (2000).

${ }^{2}$ La bibliografía dedicada al análisis social de las percepciones de la biotecnología aplicada a la alimentación es abundante desde los principios de los años 90, como muestran los ejemplos de Busch (1991), Hoban, Kendall (1992), Swoka (1993) o Zchendorf (1994). En España, las primeras aportaciones se referian a las diferentes aplicaciones, como muestra el trabajo de Moreno, Lemkow y Lizón (1992), o bien se incorporaban en tratamientos genéricos sobre las aplicaciones de la biotecnología, como Box (1993). Entre los análisis posteriores, por la amplitud del tratamiento del que las relaciones entre biotecnología y sociedad son objeto, hay que destacar a Muñoz (2000 y, sobre todo, 2001).
} 
se mejorase la selección de noticias y el tratamiento de los contenidos de las noticias publicadas. Lo cierto es que todos esos temas que hemos indicado se han convertido en algún momento en objeto de debate social, construido a partir de las informaciones ofrecidas por los medios de comunicación, pero muy particularmente por la prensa. Porque la prensa, a causa de sus especificidades, se acaba convirtiendo siempre en un punto de observación permanente de esos debates. A causa de su formato, la prensa hace más viable que muchos temas de diferentes ámbitos se encadenen día tras día en diferentes noticias, y por lo que a veces un tema puede tener una presencia en ese medio de comunicación, de manera continuada durante varios meses, tal y como ha sucedido con la biotecnología aplicada a la alimentación. Por ello es mucho mayor el número de noticias aparecidas sobre la biotecnología en la prensa que en otros medios de comunicación de masas, como la radio o la televisión, y también son más variadas en el contenido.

A pesar de la importancia que algunos atribuyen al tipo de mensajes emitidos por la prensa escrita, no nos vamos a ocupar aquí de buscar relaciones entre noticias de medios de comunicación y representaciones sociales, especialmente por el riesgo de caer en diferentes inconsistencias, pues, ciertamente, el grado de incidencia del entorno mediático en la generación de actitudes es bastante discutible. De hecho, sí tiene una incidencia directa en la opinión pública, considerando que ésta se interpreta con unas metodologías determinadas, pero ya hemos hablado de la distancia existente entre opinión pública y representaciones. Por el contrario, las actitudes se encuentran marcadas por un número mayor de aspectos individuales, sociales y culturales, con los que los mensajes comunicativos interactúan. Por ello, en este apartado, vamos a fijar simplemente cuál ha sido el espacio que ha ocupado el debate social sobre OGM en la prensa española, a fin de señalar cuándo éste ha tenido una importancia significativa para la opinión pública de acuerdo con el momento en que realmente han estado activos los medios de comunicación en este sentido ${ }^{3}$.

La biotecnología se incorporó en un primer momento a la tendencia general de los medios de comunicación de presentar los avances científicos de manera discontinua y con desigual profundidad. Dentro de esa dinámica, aparecen de forma más o menos esporádica a lo largo de la primera mitad de los años 90 algunas noticias relativas a la manipulación genética y a sus aplicaciones, pero no son objeto de un tratamiento diferenciado de otras noticias de ciencia. Muy extraño resulta encontrar hasta la segunda mitad de esa década algún artículo o reportaje donde se presenten los avances en biotecnología desde una perspectiva

\footnotetext{
${ }^{3}$ La explicación que sigue a continuación es producto de un vaciado y análisis de prensa sistemático y exhaustivo realizado sobre tres medios de prensa españoles entre 1997 y 2001: El Mundo, $A B C$ y La Vanguardia, completado con un análisis no exhaustivo de los años anteriores y posteriores, así como de otros medios de prensa de ámbito nacional y regional.
} 
de debate sobre sus oportunidades y sus riesgos. Son en cambio otros medios más específicos, como las publicaciones de entidades ecologistas y, evidentemente, las publicaciones científicas de divulgación, las primeras en abordar ese tema desde esa perspectiva y no será hasta después de 1995 cuando las noticias sobre aplicaciones alimentarias de la biotecnología se orienten hacia la exposición de un debate sobre sus beneficios y riesgos, paralelamente a un debate que crecía en intensidad en otros países europeos y que se vería promovido por una interacción entre diferentes agentes con opiniones y posiciones enfrentadas, de los cuales la prensa se hará amplio eco. El debate va a ganar intensidad progresivamente durante la segunda mitad de la década de los 90 , una vez que de los debates sobre la tecnología se pase a la discusión sobre la regulación de esas aplicaciones en el ámbito de la UE, pero no alcanzará su mayor repercusión en los medios españoles hasta el periodo comprendido entre el año 1999 y el primer tercio del 2000, momento a partir del cual se iniciará un retroceso paulatino de la presencia de noticias sobre biotecnología aplicada a la alimentación, hasta convertirse en un contenido eventual durante el año 2001 y, aún más, durante los años sucesivos. Puede decirse que, a partir de la segunda mitad del 2000 , la polémica se agota en los medios de comunicación, aunque pervive - y de qué manera - entre los agentes promotores del debate.

Vamos a dedicar algo de espacio a comentar cuáles fueron los diferentes episodios e informaciones que dieron contenido a la polémica en los años 1999 y 2000. Si bien la autorización en 1996 de comercializar los OGM abre el periodo de tratamiento específico de la biotecnología aplicada a la alimentación en la prensa española, sólo se expresa en un seguimiento eventual hasta 1998. Aun así, cabe destacar que en 1998 tiene lugar la primera cosecha de maíz transgénico en España, a través de dos variedades distintas propiedad de la empresa Novartis. En ese año aparece también una de las noticias que más eco tendrá en el desarrollo de la polémica. En agosto, se publica un estudio de Arpad Puztai, donde se indicaba que se había detectado daños en el sistema inmunológico y dificultades de crecimiento en ratas que habían comido patatas modificadas genéticamente para combatir las plagas de insectos. Este experimento será el primer gran argumento en el que se apoyarán los detractores de los OGM para aumentar su presencia crítica en la prensa, marcando, así, el despegue de la presencia de las noticias sobre alimentos transgénicos en los medios de comunicación. Ya en 1999, los medios de comunicación se hacen eco de campañas como la de Ecologistas en Acción, campañas contra el consumo de alimentos transgénicos, que se añadieron a las ya iniciadas por Greenpeace y Vida Sana y que, hasta entonces, habian tenido un eco puntual. Un mes después se publica en España el número de The Ecologist dedicado íntegramente a los alimentos transgénicos, que, de acuerdo con las versiones difundidas, no se pudo publicar en Gran Bretaña. Objeto de polémica fueron también las pruebas experimentales de nuevos cultivos, que de 36 en 1996, pasaron a 124 en 1998. A ello se añadirá la incertidumbre en las cifras 
de superficie cultivada en España con maíz transgénico, que se calculó oscilaba entre 12.000 y 20.000 hectáreas, hasta que en mayo se anunció que eran 19.000, pero sin que se supieran que áreas habían sido plantadas, ya que la adquisición de semillas tenía lugar entre los agricultores y la empresa Novartis.

En ese periodo también se recogen las primeras novedades legislativas sobre OGM. El 1 de enero de 1999 entró en vigor una normativa europea que obligaba a etiquetar aquellos alimentos que contuvieran más de un $2 \%$ de organismos modificados genéticamente, ante lo cual en febrero de ese año el Instituto Nacional del Consumo declaró ignorar si esta medida se estaba aplicando al cien por cien. En febrero hubo además una primera modificación legal europea reguladora del uso de organismos transgénicos, con el objetivo de endurecer la autorización para su comercialización. También en ese mes tiene lugar la Cumbre de Cartagena (Colombia), donde 170 países se reúnen para aprobar el Protocolo de Bioseguridad. Finalmente, esa cumbre acabaría fracasando por la obstaculización de esos siete países a imponer trabas al comercio de OGM. En los primeros meses de 1999 se publican también muchas noticias internacionales sobre polémicas asociadas a los OGM, sobre todo en Gran Bretaña.

La vida política española no era ajena esos años a un creciente clima de debate. Así, diferentes partidos y organizaciones (PSOE, Ecologistas en Acción, etc), critican que el gobierno del Partido Popular no presentara una posición clara sobre los alimentos transgénicos. Además, sindicatos como $\mathrm{CCOO}$ piden una moratoria en la liberación al medio ambiente de los OGM y en la comercialización de los mismos, como en aquel momento sucedía en Austria y Luxemburgo. Al mismo tiempo, Iniciativa per Catalunya y Nueva Izquierda interpelan al gobierno a un debate sobre la autorización del cultivo de OGM en España y critican la autorización de dos variedades de maíz y su preocupación por los cultivos transgénicos con genes de resistencia a antibióticos, ante el riesgo de que éstos se vuelvan ineficaces. Y una proposición no de ley de Izquierda Unida para prohibir durante tres años la importación, venta y cultivo de productos transgénicos en España y obligar al etiquetado, es rechazada por la Comisión de Medio Ambiente del Congreso de Diputados. En primavera empiezan a aparecer noticias referidas a medidas políticas nacionales en relación con la cuestión, y el gobierno propone en el senado la elaboración de una Ley General sobre Alimentación, mientras que esta cámara convoca una comisión de estudio ante la que comparecerán diferentes expertos. En junio se trasladará también el debate al Congreso de los Diputados. Por su parte, el gobierno vasco decreta una moratoria a la producción de OGM en su territorio. A ello se añadirá a lo largo de ese año el establecimiento de una moratoria de facto al otorgamiento de autorizaciones a nuevas aplicaciones de OGM para su desarrollo comercial.

Las numerosas noticias generadas alrededor de la cuestión no pasan desapercibidas a los agentes interesados en el desarrollo de la tecnología, particularmente científicos, y así lo expresa su presencia en diferentes artículos de opinión que aparecen con frecuencia en uno u otro diario, generalmente manifestando una 
posición favorable. Más globalmente se dan intervenciones públicas de científicos o instituciones, nacionales y europeas, dirigidas a relativizar los riesgos detectados o proclamados de esos alimentos, en particular, cuestionando los resultados del estudio de Putszai. Esa posición será ratificada en España a lo largo de esos meses por algunos de los científicos que comparecerán en el Senado.

Ya a finales de enero del año 2000, se desarrollará la Cumbre de Montreal (Canadá), donde finalmente se firmará el Protocolo de Biodiversidad. En los primeros meses de ese año se alternan diferentes tipos de noticias, de contenidos similares a los del año anterior: en marzo, la OCDE reclama un comité de análisis internacional de los transgénicos, mientras que la OMS "alerta sobre la falta de información". Por su parte, la entidad ecologista Amigos de la Tierra lanza su propia campaña contra los OGM. Algunos medios recogen también la reclamación del desarrollo de la tecnología por parte de países del Tercer Mundo. Unas de las más recogidas serán las polémicas relacionadas con los cultivos y sus impactos: en mayo, se alerta de que se han sembrado por error en Europa numerosas hectáreas con colza transgénica y que un $15 \%$ del maíz europeo puede haber sido "contaminado" con maíz transgénico. Sin embargo, la pérdida de intensidad de noticias sobre alimentos transgénicos respecto al año anterior es un hecho. La podemos comprobar mediante la comparación, en el siguiente cuadro, del número de noticias y artículos de opinión asociados a aplicaciones alimentarias de los OGM, presentes en las ediciones nacionales de tres medios de información españoles:

Tabla 1.

Comparación del número de noticias aparecidas en prensa sobre OGM entre 1998 y 2000.

\begin{tabular}{lccc}
\hline & 1998 & 1999 & 2000 \\
\hline ABC & 3 & 26 & 34 \\
EL MUNDO & 5 & 59 & 38 \\
LA VANGUARDIA & 3 & 46 & 27 \\
TOTAL & 11 & 131 & 99 \\
\hline
\end{tabular}

Dado que no disponemos de un seguimiento igual de detallado para los años posteriores, no aportaremos cifra alguna, pero sí queremos hacer notar que un seguimiento no exhaustivo de la cuestión durante los años 2001, 2002, 2003 y 2004 ha revelado una baja presencia de las noticias sobre aplicaciones biotecnológicas. Ello se ha concretado en un menor número de noticias noticias y en una menor extensión de las mismas, respecto a los años 1999 y 2000 , pero superior a 1998 . Y todo ello, sin que hayan faltado oportunidades para un resurgimiento público de la polémica, tanto en el periodo 2002 y 2003, cuando se debatían nuevas directivas europeas, como en mayo de 2004, cuando la autorización de una nueva variedad de maíz (llamada Maiz BT-11) rompía la moratoria aplicada durante seis años. 
RIS

REVISTA INTERNACIONAL DE SOCIOLOGIA

№ 40 , ENER0-ABRIL, 2005

ELENA ESPEITX Y JUAN JOSÉ CÁCERES

Una vez mostrada esta evolución, vale la pena destacar dos aspectos. Por un lado, que la presencia de los OGM en la prensa sigue un patrón ajeno a su discusión en las instituciones, donde en modo alguno ha disminuido después de 2001 y, por otro, que ese patrón responde a un ciclo informativo que, desde una manifestación leve, experimenta un crecimiento rápido, seguido de un retroceso paulatino, hasta retornar a los niveles anteriores a 1998. En otras palabras, la propia prensa incrementa las noticias sobre OGM cuando desea fijar su debate como un tema de actualidad, y les da de lado cuando decide que deje de serlo.

\section{LA LÓGICA DE LOS AGENTES}

Hemos dedicado gran atención a los medios de comunicación porque son el ámbito mediador privilegiado para la circulación de la información entre los agentes y el resto de la sociedad, el foco sensibilizador del ciudadano, el terreno donde todo agente social con ánimo de incidir sobre la opinión pública tiene que llegar. Ese ánimo se vuelve verdadera obligación para los agentes cuando un debate se enciende, es decir, cuando los medios de comunicación son la arena del combate entre posiciones confrontadas, cuyo resultado puede decidirse en función de la información recogida por los sistemas de estudios de la opinión pública. Todos conocen bien la importancia de los medios de comunicación para la formación de la opinión, aunque, sin duda, saben bastante menos de los sentidos que la opinión pública cobra en el verdadero terreno social ${ }^{4}$.

Los agentes buscan convencer y con esa lógica pretenden hacer hegemónicos su mensaje en los medios. Sabiendo que en esta cuestión concreta la actitud y el rechazo de los OGM puede frenar el desarrollo de estas tecnologías, "formar" $\mathrm{y}$, sobre todo, "convencer" se considerará una necesidad. Para muestra, las campañas publicitarias llevadas a cabo por una multinacional como Monsanto o una entidad ecologista como Greenpeace, ambas a finales de los años 90. Pero no solamente estas entidades. También los científicos que trabajan en este campo se han lanzado a una campaña de "formación" para defender su ámbito de investigación - dicho sea, sin el menor asomo de crítica, que esta defensa puede muy bien corresponder a la convicción sincera de que los beneficios pueden ser muy superiores a los riesgos-. Un análisis de los medios de comunicación muestra que un cierto número de expertos aparecen de manera recurrente en todo tipo de foros para defender las aplicaciones biotecnológicas. También se evidencia que

\footnotetext{
${ }^{4}$ Mucho rubor generan determinadas interpretaciones de los agentes sobre el comportamiento ciudadano, convertido en consumidor para la ocasión en lo que se refiere a los OGM, dando así mucho espacio a los científicos sociales, si éstos juegan a la interpretación social, en lugar de dar juego y sustento a la opinión interpretada.
} 
éstos conocen los argumentos de los oponentes al dedillo y que construyen sus contrargumentos a partir de éstos, y viceversa. Así, los elementos que aparecen en el debate se construyen dialógicamente a medida que éste se desarrolla. Ningún argumento es gratuito - responde a unos contrargumentos-, ni banal - utiliza determinados valores, interpela determinadas sensibilidades-. Las estrategias persuasivas y las argumentaciones se construyen a partir de la idea que los diferentes agentes tienen de los posicionamientos de los consumidores y de las vías que parecen más efectivas para convencerlos. Pero no son sólo estos tres agentes (científicos, periodistas o expertos en opinión, y ciudadanos) los que participan en este proceso. El espacio de la comunicación convoca agentes sociales diversos: miembros de la administración, representantes de partidos políticos, miembros del gobierno, científicos de diversas disciplinas, representantes de la industria y del mundo empresarial, sindicatos, asociaciones diversas, intelectuales, tertulianos, periodistas..., y la misma ciudadanía, a través de los espacios abiertos a su participación (cartas de los lectores, participación directa en programas de radio o de televisión que permiten esa posibilidad, etc.). No debe olvidarse, sin embargo, que la voz de los ciudadanos en los medios de comunicación no aparece en las mismas condiciones que la del resto de los agentes. La mayor parte de las veces no aparece directamente y ocupa un espacio reducido en relación con el resto de voces que se manifiestan en el debate. No obstante, los ciudadanos - 0 los consumidores, porque es en estos términos como se les nombra y en calidad de tales como se les interpela la mayor parte de las veces, aunque no sea ésta la condición que uno tiene cuando ejerce de opinión pública - tienen un papel relevante en la información que circula en los medios de comunicación. Los discursos se construyen en "su honor", les están destinados, están pensados para convencerlos. Los diferentes agentes compiten entre sí en el intento de inclinar la opinión de los ciudadanos en su favor y captar su atención.

Mostrado el papel de los agentes, hay que añadir que los medios de comunicación no se limitan a transmitir una información neutra -ni en la información política, ni en la deportiva, y tampoco, como es obvio, en la científica-. Vehiculan y transmiten mensajes de los diversos agentes - a menudo contradictorios, puesto que defienden intereses diferentes-, pero no solamente. Conscientes o no de su papel, son un agente activo más al construir la noticia, una noticia que se construye al elegir los temas a tratar, al escoger los informantes a los que se va a dar voz y al decidir qué sentido se da a todo ello. La dispersión de los focos de investigación y la mezcla de voces que aparecen para defender intereses diversos, cada cual con su propia legitimidad interpelando de diferentes maneras a los ciudadanos, convierten la supuesta "divulgación" de las noticias científicas en un debate. En efecto, los propios medios de comunicación completan con su propia lógica una evolución de la confrontación de posiciones al debate social, y es en ese momento cuando empieza a cobrar importancia la opinión del ciudadano, o mejor dicho, cuando ésta se convierte en opinión pública. 


\section{BUSCANDO EXPLICACIONES AL RECHAZO SOCIAL DE LOS OGM}

El periodo de alta presencia mediática de los OGM coincidió con resultados adversos en las encuestas sobre la opinión pública respecto a su desarrollo y con la consolidación de una moratoria "de facto" en la autorización del cultivo de nuevas variedades en los campos europeos. Ello propició el establecimiento de numerosas causalidades tendentes a relacionar decisiones institucionales, opinión pública interpretada y rechazo de los OGM por parte de la población europea. Las explicaciones sobre ese rechazo se dirigían hacia la actitud y el papel interpretado por los oponentes y los comunicadores, pero se fundamentaban en algunas ideas significativas, que se han ido reiterando en los últimos tiempos. Vamos a observar ahora algunas explicaciones que han aparecido recurrentemente en el debate social para argumentar ese rechazo, para conocer cómo operan en las representaciones sociales. También nos ocuparemos de cómo operan algunos argumentos utilizados por los detractores para observar hasta qué punto penetran en las representaciones individuales 5 .

\section{¿La ciencia en entredicho?}

Cuando se aborda la cuestión de las "representaciones" sociales alrededor de las aplicaciones de la ingeniería genética, se hace referencia a menudo a las valoraciones sociales de la ciencia. Se llega a hablar de "cienciofobia" por parte de determinados sectores de la sociedad, que se opondría a la "cienciofilia" de otros. El debate sobre los OGM debería interpretarse, desde esta perspectiva, en las oscilación entre estos dos polos. En las valoraciones negativas - las que alimentarían la cienciofobia - influirían tanto los debates internos de las llamadas "ciencias duras", como las reflexiones críticas por parte de las ciencias sociales, en particular, de la sociología de la ciencia. En las valoraciones positivas - que alimentarían la cienciofilia-, pesarían sobre todo los "logros" de la ciencia, su potencial de "progreso" y mejora en la calidad de vida. Sin embargo, conviene hacer algunas precisiones.

Por lo que respecta a las valoraciones negativas de la ciencia, habría que señalar, en primer lugar, que los debates internos de las disciplinas científicas y los análisis críticos de las ciencias sociales tienen escasa repercusión fuera de sus ámbitos concretos respectivos, y el debate entre reduccionismo y holismo, o las

\footnotetext{
${ }^{5}$ Los siguientes apartados se han elaborado a partir de los resultados del trabajo de campo desarrollado en el proyecto, basado en dos técnicas de análisis cualitativas: las entrevistas personales y los grupos de discusión. Se trata de enfoques empleados también con éxito en estudios internacionales, como por ejemplo Marris, Wynne, Simmons y Weldon (2002). A la información recogida en ese trabajo corresponden las citas que se incluyen en el texto.
} 
reflexiones postmodernas sobre ciencia y sociedad no están en el centro de las preocupaciones de la mayor parte de la población. En segundo lugar, que los ciudadanos no suelen poner en entredicho la ciencia en general y "en mayúscula", ni el método científico, ni, y esto es importante destacarlo, las explicaciones del mundo que aportan actualmente las diferentes disciplinas científicas; entre otras cosas, porque estas explicaciones penetran escasamente en las representaciones sociales sobre la mayor parte de ámbitos en los que la ciencia proporciona explicaciones. Las perspectivas de la física cuántica, por poner un ejemplo, inciden muy poco en la percepción intuitiva del mundo de la mayor parte de los individuos. No son los "conocimientos" científicos, sus saberes y explicaciones sobre el mundo, lo que se pone en entredicho - o se valora muy positivamente-de la ciencia, sino sus aplicaciones técnicas, y su poder transformador de la vida cotidiana. Lo realmente decisivo en la percepción social de la ciencia es su enorme efectividad, que resulta de la unión entre conocimiento, tecnología y recursos económicos, con sus consecuencias, percibidas claramente, y consideradas a veces positivas, a veces negativas. Se puede confiar o desconfiar de estas aplicaciones, pero se cree en ellas. Y hablamos de creer en la ciencia, no de conocer, porque en este ámbito, como en tantos otros, las actitudes se basan en gran parte en creencias, no en conocimientos bien fundamentados. En términos generales, la ciencia - como forma de cocimiento con capacidad de incidir en la realidad - se valora positivamente.

Por otro lado, es preciso no confundir la valoración de la ciencia y sus aplicaciones, con la valoración de los "científicos". Normalmente, no se cuestiona -salvo en casos puntuales-el método científico y su resultado en conocimientos, ni tan sólo sus aplicaciones en muchos casos, sino a los científicos como individuos. Y para valorar a los científicos como individuos, se parte de la experiencia personal y de las representaciones culturales y sociales sobre cómo se comportan estos individuos en sociedad, qué estrategias adoptan, qué motivaciones e intereses mueven sus acciones.

Sin embargo, admitiendo esta premisa de que no hay una mayoritaria actitud negativa ante la ciencia, se imponen algunas matizaciones. Una actitud ampliamente compartida ante la tecnología es que ésta, aun siendo fuente de progreso, puede conllevar también efectos indeseados. La valoración, en principio positiva, puede verse, después matizada al introducirse la idea de "los fines" a los que se destine, y eso es algo que los OGM ponen de manifiesto. Esta consideración lleva a algunos a afirmar que, aun siendo, en principio, ciencia y tecnología positivas, los fines no siempre son deseables o, como mínimo, los más convenientes. Además, añaden otros, si no se destina a buenos fines, es porque está al servicio de los intereses económicos. A pesar de que la mayor parte de las reflexiones sobre ciencia y tecnología se basa en la distinción que se suele establecer entre ciencia y sus aplicaciones, que es la que introduce matices, también hay posiciones más tajantes. Así, hay quién considera la ciencia como un ente al que no debe oponérsele ningún freno, casi omnipotente en su capacidad de incidir sobre 


\section{RIS}

el mundo, y capaz de resolver incluso los problemas que ella misma genera. Pero también hay quién le atribuye los peores efectos, quién afirma que ciencia y tecnología "están estropeảndo el mundo". Debe destacarse, sin embargo, que estas posiciones más extremas, de fe inquebrantable en la ciencia o de absoluto rechazo, son minoritarias. Las actitudes más frecuentes oscilan entre una valoración muy positiva de algunos aspectos de la tecnociencia, las reservas ante unos y la indiferencia ante otros. Así, la actitud ante las innovaciones tecnológicas despierta actitudes ambivalentes. En principio se suelen considerar mejoras, y se valoran, pues, positivamente. Sin embargo, también se afirma que cualquier mejora comporta algún efecto indeseado o algún riesgo. En otras palabras, que todo progreso tiene un precio, una contrapartida. Esta constatación raramente lleva a la conclusión de que deban evitarse las innovaciones por principio, y que, en términos generales, se consideren mayores las ventajas que los inconvenientes. Ahora bien, a causa de ese precio a pagar, de esa contrapartida, también aparece de manera recurrente la idea de que es preciso controlar la tecnociencia. Sobre esta necesidad de control, y sobre quién debe ejercerlo, volveremos más adelante. Esta actitud ambivalente ante la ciencia, en general, es la misma que se encuentra ante los OGM más que tajantes rechazos o entregadas aceptaciones predomina el reconocimiento simultáneo de dimensiones negativas y positivas.

\section{¿Miedos irracionales?}

En el debate alrededor de la biotecnología, algunos de los agentes implicados aluden al miedo de los ciudadanos como un factor negativo que actúa de freno a los avances, al progreso. A menudo se tacha ese miedo de irracional, surgido de la ignorancia; para algunos, del oscurantismo.

Es cierto que se detectan temores, miedos más o menos difusos, lo cual no es sorprendente si se considera que el ser humano tiene la capacidad de proyectarse en el futuro, de plantearse los riesgos, no sólo para uno mismo, sino también para las generaciones futuras. Se teme lo que pueda suceder mañana a uno mismo y a los próximos, y esto incluye diferentes amenazas. En primer lugar, las amenazas no identificadas, que por desconocidas son imprevisibles y, por tanto, despiertan temor. Porque todo aquello que es imprevisto puede tener efectos positivos o negativos, y si los positivos se desean, los negativos se quieren evitar. Y ante las aplicaciones tecnocientíficas se puede sentir este tipo de temor. Pero no sólo; también hay toda una serie de amenazas perfectamente identificadas. No se teme únicamente lo desconocido, se teme también aquello que se conoce y se sabe que entraña problemas o sufrimiento. El riesgo en este caso es concreto y tiene nombre, puede llamarse cáncer, Alzheimer o paro. En cualquier caso, los temores no son reducibles a miedo a la ciencia o la técnica, sino que el ámbito de lo cuestionado es más amplio, e incluye los fines de las aplicaciones tecnocientíficas y los resultados. Esto no debe olvidarse, porque precisamente la cuestión de los 
fines y los resultados obliga a preguntarnos si no es mayor -0 , como mínimo, igual de persistente- el miedo a lo viejo conocido, que a lo nuevo desconocido. Es decir, cabe preguntarse si una buena parte de los temores no provienen de experiencias pasadas, que demuestran que lo peor a veces sucede, y que lo menos bueno es frecuente. Así se manifestaba un informante de mediana edad: "yo me imagino que el principal problema son todos los fallos humanos que ha habido. Muchas cosas se han vuelto contra nosotros, y esto de los transgénicos podría ser uno más."

\section{Formación e información: ¿la panacea?}

Para aquéllos que consideran que el supuesto rechazo social a los OGM es fruto de la ignorancia y el miedo, éstos son los enemigos que deben combatirse, y el medio para ello es el conocimiento. Formación e información se convierten en instrumentos necesarios y suficientes. Para conseguir introducir fácilmente nuevas tecnologías bastaría con arrancar los elementos irracionales y contradictorios que condicionan su percepción, y para ello haría falta una estrategia eficaz de educación científico-técnica.

Esto, aunque puede ser en parte verdad, merece ser discutido. Es cierto que la mayor parte de los ciudadanos tiene escasos conocimientos precisos sobre las dimensiones científicas de estas técnicas y sobre el desarrollo de la investigación o la reglamentación, pero esta falta de conocimientos no explica todos los rechazos o todas las aceptaciones de las biotecnologías agrícolas. Por un lado, y en contra de lo que se afirma recurrentemente sobre la aceptación o rechazo de los OGM, el conocimiento no es ni condición necesaria ni suficiente. La consideración de que "todo lo nuevo encierra posibilidades desconocidas, buenas y malas", es un factor a considerar, pero no actúa siempre con tanta virulencia, ni por fuerza en el sentido del rechazo. Es verdad que al principio muchas innovaciones producen reticencias, reservas, y se especula sobre las posibilidades negativas, pero no llegan a generar un debate ni llevar a claros posicionamientos en contra. No se conoce la mayor parte de las tecnologías que son centrales en la vida cotidiana - y mucho menos los conocimientos científicos en los que se basan-y eso no genera ningún conflicto. Por eso, el desconocimiento sobre las bases de una innovación tecnológica no implica necesariamente la generación de un debate sobre su caso. Pero tampoco es un factor suficiente, porque el conocimiento, en caso de existir, no garantiza la aceptación o el rechazo simplemente porque tal aceptación o rechazo se fundamenta en otros elementos que nada tienen que ver con el estricto conocimiento de los fundamentos científicos de la técnica. El

${ }^{6}$ Cita extraída de un grupo de discusión y correspondiente a una mujer de 28 años. 
RIS

REVISTA INTERNACIONAL DE SOCIOLOGÍA

№ 40, ENERO-ABRIL, 2005

ELENA ESPEITX Y JUAN JOSÉ CÁCERES

nivel de formación es una variable que hay que tener en cuenta, pero no la única variable con potencia explicativa. Deben tenerse en cuenta otros factores, como los estilos de pensar, la visión que se tiene de la naturaleza y del lugar que ocupa el ser humano en el mundo, los posicionamientos ideológicos y políticos, el enfoque intelectual o disciplinario, las experiencias previas y el lugar que se ocupa en el espacio social... En cualquier caso, la falta de información y de formación no se traduce mecánicamente en rechazo ante las innovaciones científicas, aunque se observen ligeras diferencias porcentuales en los eurobarómetros. La actitud de confianza plena, de total entrega a ciencias y científicos, es más frecuente entre aquéllos que tienen una formación científica muy elemental. El desconocimiento se puede traducir así en una vía que facilita la aceptación, más que el rechazo. Consideraciones sobre, por ejemplo, las presiones y los intereses que subyacen a la investigación científica, aparecen más a menudo entre individuos con un elevado nivel de formación, mientras que la fe en "lo que se hace en el laboratorio" es más frecuente entre los que no disfrutan de ésta. Con esto no se quiere decir que sea deseable el desconocimiento para el avance tecnocientífico, sino que la actitud crítica se ve a veces favorecida precisamente por una mayor información.

Por otro lado, a veces se intenta explicar como miedo ante la ciencia o la innovación, lo que en realidad es desconfianza en quienes se considera deben controlar sus aplicaciones, es decir, en las instituciones, aunque el grado de interiorización de dicha desconfianza pueda variar mucho de un individuo a otro. Algunas experiencias recientes y pasadas - el aceite de colza forma parte de la memoria colectiva - avalan esta percepción. La incompetencia o la mala $\mathrm{fe}$ - según la idiosincrasia del individuo-, que se achacan a la instituciones en el caso de la crisis de las "vacas locas", se les atribuye a la hora de valorar otras actuaciones, entre ellas, la regulación de los OGM. La desconfianza no surge a raíz de los OGMs, pero șe aplica por analogía. Así, aunque pueda no existir una real desconfianza hacia la tecnología en cuestión, se puede desconfiar absolutamente de aquellos que la van a gestionar, a dar sentido y a imprimir una dirección. Lo mismo puede decirse de las reservas que despiertan las empresas que van a sacar beneficios directos de la biotecnología.

\section{Todas las aplicaciones no son iguales}

Se detecta en los estudios que los ciudadanos no valoran del mismo modo la biotecnología aplicada a la producción agroalimentaria, que sus aplicaciones médicas. En efecto, esta distinción se establece claramente. No cabe duda que la salud es considerada central para obtener calidad de vida. Nadie considera que gozar de un estado óptimo de salud sea irrelevante; casi todo el mundo teme al sufrimiento y a las minusvalías, puesto que la enfermedad es uno de los grandes temores vigentes en nuestra sociedad. También genera angustia el envejecimiento y la pérdida de facultades. Además, en nuestro contexto social la salud y la 
longevidad son consideradas casi un derecho exigible, aunque muy a menudo no se concrete. $\mathrm{El}$ acceso más o menos generalizado a la sanidad pública, las condiciones higiénicas y de salubridad y la alimentación asegurada - aunque socialmente diferenciada - , parecen garantizar unos mínimos de salud, aunque luego vengan las cardiopatías y las neoplasias a destruir expectativas. No resulta, pues, sorprendente, en este contexto, que se valoren muy positivamente los avances en medicina que prevengan o curen enfermedades, o que prometan hacerlo en el futuro. Incluso los detractores acostumbran a reconocer ventajas a la insulina recombinante. Por ello, las promesas de la biotecnología en este ámbito son bien recibidas mayoritariamente.

¿Qué sucede con las aplicaciones agroalimentarias? Es evidente que la alimentación es una necesidad básica, y que satisfacer esta necesidad es condición necesaria para gozar de buena salud. Pero también es evidente que esta necesidad está ampliamente satisfecha en nuestra sociedad. La diferencia fundamental entre la insulina recombinante y el tomate flavr savr ${ }^{7}$ reside en su necesidad y utilidad. La necesidad de la insulina para el diabético es conocida, y también que la tecnología recombinante permite conseguirla con mayor facilidad y seguridad, pero la necesidad del nuevo tomate no se percibe con la misma acuidad. Esto ha llevado a afirmar que la diferencia estriba en la distinción entre bien individual (la salud) y bien social (la producción alimentaria). Esta distinción no parece en absoluto evidente. La "utilidad" de los avances médicos es percibida positivamente, tanto para el individuo como para la sociedad. Cuando se presenta los OGMs como soluciones a problemas sociales, la percepción de utilidad mejora las valoraciones, no las empeora. Para algunos todo aquello que sirva para aumentar la producción alimentaria es bienvenido, pero también lo son las aplicaciones que puedan servir para resolver problemas en zonas difíciles. Simplemente, lo que ocurre es que no se percibe en nuestro entorno, en general, ni útil ni necesaria esta tecnología, ni social ni individualmente.

\section{De posibilidades y barreras: los límites de la ciencia}

La cuestión de los "límites" de la ciencia es un tema de fondo que recorre el debate entre los agentes y que se expresa también en representaciones y actitudes de aquéllos que no participan en él. De hecho, no se trata de una cuestión, sino de dos: en primer lugar, de si puede o debe ponerse algún límite a la ciencia. Y en segundo lugar, y en caso afirmativo, de cómo hacerlo. Si no se alcanza consenso entre los agentes en el debate, tampoco se alcanza fuera de él, pero se plantea también, en cierto modo, en los mismos términos. Tanto para algunos agentes

\footnotetext{
${ }^{7}$ Variedad de tomate modificada genéticamente de larga conservación comercializada en Estados Unidos desde 1994.
} 
del debate, como para algunos que no lo son, no es deseable, acaso tampoco posible, establecer cuáles han de ser los límites del conocimiento científico y de su aplicación. En primer lugar, porque su desarrollo se justifica y se legitima por sí mismo, o incluso no precisa legitimación. Ésta no es, sin embargo, la actitud más extendida. También los hay que consideran, no sólo que no se deben poner límites, sino que advierten que ello es imposible. Por un lado, porque se parte del supuesto de que la ciencia se enmarca en una dinámica propia, con un impulso que la dirige quién sabe dónde, pero que, en cualquier caso, no depende de la voluntad colectiva. Como mucho, de la voluntad - y la sabiduría - de unos cuantos escogidos que sabrán advertir de las consecuencias indeseadas. Los frenos extracientíficos son considerados meros obstáculos que se acaban salvando, que deben salvarse. Por otro lado, porque se supone que los límites son imposibles de establecer ya porque nadie está capacitado para ello; en este caso, la ciencia es aún más reificada, está en sus propias manos, es dueña de su destino. La única intervención posible consiste en reparar los posibles efectos perversos. Aprender de los errores es la máxima, ya que nadie puede predecir, nadie puede limitar: "No hay que poner límites, hay que aprender de los errores. Si no baja Dios y nos dice que de ahí no se puede pasar - y no va a bajar - tenemos que aprender de los errores." 8 Dentro de esta perspectiva, la actitud más "tibia", la que hace de puente con otros puntos de vista, es la que propugna que no hay que poner límites a la ciencia, que debe dejarse libre su desarrollo, pero manteniendo una actitud vigilante. Ella marca la ruta, nosotros vigilamos que no atropelle a nadie. O si lo hace, haciendo el menor daño posible.

Pero esta actitud no es unánimemente compartida. Aun pensando que la tecnociencia es fuente de beneficios y de progreso, también hay quién teme sobrepasar unos límites. El principal problema sería el no saberse a ciencia cierta -y nunca mejor dicho- dónde puedan o deban estar tales límites. A aquellos que consideran que sí debe decidirse hasta dónde puede llegar la tecnociencia, y qué umbral no se puede traspasar - y éstos sí distinguen entre ciencia y aplicaciones-, se les plantea entonces un problema: ¿con qué criterios se establecen los límites? Y, sobre todo, ¿qué clase de criterios?: ¿criterios científicos y técnicos?, ¿criterios morales?, ¿criterios políticos?, ¿criterios económicos? Aunque en la vida real estos dos últimos criterios son los que prevalecen, en el ámbito no de cómo se actúa, sino de cómo se piensa el mundo, los criterios que deben regir las elecciones no están claros. Para algunos, los únicos criterios válidos y fiables son los científico-técnicos. Sólo desde de la perspectiva de la ciencia y la tecnología, sólo a partir de los conocimientos sobre ciencia y tecnología, se puede valorar dónde acaba el suelo firme y dónde empiezan los terrenos pantanosos

\footnotetext{
${ }^{8}$ Hombre, 36 años.
} 
de la incertidumbre. Para otros, la ciencia ni puede ni debe marcar hasta dónde llegar. En primer lugar, porque la incertidumbre es ineludible aun partiendo del conocimiento científico-técnico. En segundo lugar, porque se considera que los criterios deben ser ante todo morales. Lo cual plantea otro problema: ¿quién decide qué criterios morales deben imponerse? Ahí ya entran los posicionamientos de cada cual, las ideologías, las creencias; un terreno difícil para dirimir debates, para zanjar cuestiones, para establecer límites, terreno en el que pueden surgir -y surgen - muchos desacuerdos.

\section{De la imprevisibilidad de sus consecuencias}

En los argumentos de los oponentes suelen salir referencias a la imprevisibilidad, a la incertidumbre, que se relacionan estrechamente con la cuestión de los límites y que se aplica sobre todo a los efectos sobre el medio ambiente. Incertidumbre en el sentido de carencia informativa, que se traduce en la imposibilidad de prever todas las posibilidades e implicaciones, todos los efectos. Sobre esta cuestión también encontramos algunas coincidencias entre argumentos de los agentes y reflexiones de ciudadanos no implicados. En el caso de los OGM, los oponentes destacan que el problema radical de la incertidumbre reside en la imposibilidad de volver atrás después de la liberación de tales productos en el medio ambiente. Estos argumentos se encuentran también integrados en las representaciones sociales. Es significativo que este argumento haya sido oído, interpretado e interiorizado en la población, cuando un gran número de argumentos - en particular, los sanitarios - no parecen haber hecho tanta mella en los receptores de los mensajes. Algo nos indica sobre las inquietudes medioambientales de amplios sectores de la población. La imprevisibilidad se aborda, tanto por los oponentes como por los ciudadanos, desde la analogía, fortaleciendo la percepción de que riesgos anteriormente no previstos, quizás no previsibles - que no es lo mismo-, se acabaron concretando en efectos indeseados: "A veces hay riesgos que no se conocen hasta mucho más tarde, como aquello de la talidomida".

\section{De la producción agraria y sus problemas}

La producción agraria y sus problemas es una cuestión que despierta también actitudes ambivalentes, y que debe tenerse muy en cuenta porque constituye el marco en el que se sitúan y se interpretan los OGM. Esta ambivalencia se manifiesta en relación con el progreso en el ámbito de la agricultura y de la alimentación, de una manera más amplia. A menudo se expresan inquietudes sobre la evolución

\footnotetext{
${ }^{9}$ Mujer, 41 años.
} 
actual de toda la cadena agroalimentaria. Junto a las ventajas claramente percibidas y valoradas de la producción alimentaria actual - ventajas que van desde la abundancia y la variedad hasta la comodidad, la accesibilidad y el servicio incorporado-, se evocan sus desventajas e inconvenientes; inconvenientes que giran, sobre todo, alrededor de la pérdida de sabores y la contaminación de la agricultura. A pesar de una satisfacción global ante el estado natural de la alimentación, se expresan, pues, reservas sobre los beneficios potenciales de una industrialización creciente de la cadena agroalimentaria. En ese contexto, los OMG son considerados una etapa más en una evolución percibida ya como excesivamente avanzada. No se suelen establecer diferencias claras entre "nuevas tecnologías agrícolas" y "tecnologías propias de la Revolución Verde", sino que se perciben como formando parte de un mismo modelo.

\section{El mito del riesgo cero}

Ésta es una de las cuestiones que aparecen hasta la saciedad en los argumentos de los agentes del debate, y que está ausente en las reflexiones del resto de la población. A nadie se le ocurre - a casi nadie - pensar que el "riesgo cero" existe, si no es en un mundo ideal. En general, los ciudadanos-consumidores no exigen "riesgo cero", ni la certeza absoluta de que los OMGs no vayan a tener ningún tipo de impacto. Se tiene conciencia de que en la vida cotidiana un gran número de actividades suponen algún tipo de riesgo, que se suelen asumir poniendo en una balanza los riesgos y los beneficios que estas actividades representan. Lo que no se acepta tan fácilmente es incurrir en riesgos innecesarios — no es fácil determinar cuáles lo son y cuáles no-, en particular si el origen de estos riesgos es la incompetencia, la falta de escrúpulos o la avidez de otros agentes. Por otro lado, los riesgos que se asumen voluntariamente - como lanzarse desde un puente atado de una cuerda, o fumar - son percibidos de manera bastante distinta que aquellos que son el resultado de voluntades ajenas. El hecho de que se asuma que el "riesgo cero" no existe hace que se reaccione más bien con irritación cuando la administración o las instituciones responsables insisten en emitir un mensaje de "todo está bajo control", o "la seguridad está plenamente garantizada"..., más cuando se sabe que esto difícilmente es así, cuando un gran número de evidencias y la propia experiencia avalan lo contrario. Las afirmaciones emitidas por científicos, que hablan de controles y de estudios demostrando seguridad, suelen parecer más creíbles, aunque a menudo se matizan estos mensajes aduciendo que "por mucho que digáis, cada cara tiene su cruz, aunque esta tecnología suponga beneficios, siempre hay algún inconveniente, algún precio a pagar"10.

\footnotetext{
${ }^{10}$ Mujer, 33 años.
} 


\section{De ciencia y tecnología y de economía y política}

Del mismo modo que los discursos de los agentes en el debate, las actitudes de los ciudadanos ante los OMG están moldeadas por consideraciones sociales, políticas y económicas que cuestionan las fronteras entre ciencia y política. Los problemas medioambientales y las implicaciones sociales están en el centro de sus reservas, del mismo modo que son consideraciones sociales y económicas las que se arguyen desde la aceptación. Aquellos que suscriben que los transgénicos, como cualquier innovación tecnocientífica, deben impulsarse, lo hacen a partir de consideraciones sociales: "es necesario poder alimentar a toda la humanidad"11 o "si esto va a dar mayores beneficios a los agricultores, adelante"12. También aluden al atraso tecnológico, pero también económico, que supondría para los países que frenaran este desarrollo, con relación a los que le dieran un fuerte impulso. No suelen defenderlos desde la perspectiva de progreso del conocimiento ni de la ampliación de los saberes sobre el mundo. Entre aquellos que plantean reservas ante la biotecnología de manera más o menos tajante - y esto varía mucho de un individuo a otro-, tampoco las dudas se suelen centrar en aspectos puramente técnicos o científicos, sino en sus implicaciones sociales. Aun en los casos minoritarios - y esto sí que se relaciona estrechamente con el nivel de conocimientos - en que se pone en duda la tecnología misma, se acompaña de otro tipo de reservas, de cariz socioeconómico o político. Cabe preguntarse si, con un bagaje superior de conocimientos específicos, el debate se circunscribiría en mayor medida en los aspectos tecnológicos y científicos. Probablemente sí, en particular por parte de aquéllos que, por sus actitudes vitales y por sus estilos de pensar, no cuestionan el desarrollo científico ni le imputan responsabilidades en problemas sociales candentes. Para éstos, el único debate es el de la técnica y el de los conocimientos en que se basa. El perfeccionamiento de la tecnología y la exigencia de estudios serios y de controles rigurosos sería condición necesaria y suficiente, aunque cuestiones sociales, como las posibilidades de desarrollo económico y de competitividad, estarían, sin duda, también presentes. Para aquellos que sí asocian el desarrollo tecnocientífico a otros aspectos de la vida social, el mayor nivel de conocimiento científico se traduciría en incorporar, junto al debate técnico, el debate social. Es decir, para determinadas actitudes vitales, para determinados estilos de pensar, ambas cosas son indisociables. Así, en individuos con un cierto nivel de conocimientos se encuentran discursos complejos que no establecen una barrera neta entre ciencia y sociedad y que integran argumentos de distinto orden. En cualquier caso, no debe olvidarse que las consideraciones sociales, que son ideológicas y morales, están presentes sea cual fuere la posición

\footnotetext{
${ }^{11}$ Hombre, 62 años.

${ }^{12}$ Hombre, 23 años.
} 
RIS

REVISTA INTERNACIONAL DE SOCIOLOCÍA

№ 40, ENER0-ABRIL, 2005

ELENA ESPEITX Y JUAN JOSÉ CÁCERES

adoptada: a favor del desarrollo tecnocientífico (que se vincula al económico, y, por tanto, es social) o planteando dudas a determinados desarrollos, por razones también sociales. Intentar separar las diferentes vertientes de la controversia no parece tener demasiado sentido en este caso. Tenerlas en cuenta las complica sobremanera, porque en algunas cuestiones, los desacuerdos son radicales.

\section{Aspectos éticos y morales}

Junto con las preocupaciones inmediatas y los factores individuales de personalidad, de nivel de formación o de recursos, aparecen algunos temas recurrentes que atraviesan las reflexiones sobre las nuevas biotecnologías y sus implicaciones, tanto de los agentes implicados como del resto de la sociedad. No son temas que se relacionen exclusivamente con la biotecnología, ni tan sólo con la tecnociencia en general. Además de las diferentes posiciones vitales ante el papel del hombre en el mundo, de su relación con la naturaleza y de los límites de su intervención, que parecen tener una relación directa con las actitudes ante el desarrollo científico y tecnológico, hay otras cuestiones que también aparecen en el centro de muchas consideraciones. Se trata de reflexiones que se podrían catalogar como morales o éticas, y que, sin duda, también son ideológicas. Se ha comentado que en las valoraciones sobre biotecnologías aparece constantemente la referencia al buen o mal uso que se haga de ellas. Considerar bueno o malo un uso, una utilización, es un criterio moral, que se sustenta en valoraciones éticas y en posicionamientos ideológicos. Considerar, por ejemplo, que una buena aplicación de la biotecnología sería resolver el hambre en el mundo, o que un problema de esta técnica es que acrecienta las desigualdades entre norte y sur, por ejemplo, significa apelar a principios morales e ideológicos. Presupone creer en la solidaridad, en la igualdad, en la justicia. Que la justicia - la justicia universal, no la que imparten los hombres - y la igualdad no existen, es bien sabido. Como también es bien sabido que considerar que éstas son deseables y un objetivo al que tender son concepciones bien arraigadas en el ser humano; del mismo modo que está bien arraigado en el comportamiento humano vulnerar las normas que se establecen para alcanzarlas. De la misma manera que las actitudes vitales y los estilos de pensar la relación hombre/naturaleza varían de un individuo a otro - repitiéndose, sin embargo, un limitado número de posibilidades, culturalmente pautadas-, las actitudes y los estilos de pensar, respecto a grandes principios como la igualdad o la justicia no son compartidos por todos los individuos. Para unos, la igualdad o la justicia son deseables y deben ser perseguidas a toda costa y conviene mantener actitudes vigilantes para evitar todo aquello que profundice desigualdades e injusticias. Para otros, la desigualdad, la injusticia, son realidades ineludibles, inevitables, que forman parte del orden natural de las cosas, aunque el ser humano, con sus actuaciones y sus decisiones, las pueda reducir. Otros consideran, en cambio, que no sólo son ineludibles, sino que incluso son deseables, y que nada puede ni debe hacerse para evitarlas. 


\section{CONCLUSIONES}

Hemos presentado en este artículo la necesidad de diferenciar opinión pública y representaciones sociales en el conocimiento de las actitudes sociales hacia los OGM, y para ello hemos presentado, primero, la evolución del debate social y el papel de los agentes dinamizadores del mismo. A través de ello hemos visto que la controversia que se genera a su alrededor está protagonizada por determinados agentes que movilizan discursos y argumentos, que vehiculan representaciones y que consiguen transmitirlas a través de los sistemas de comunicación pública. Ese es uno de los contextos donde hay que situar las representaciones sociales respecto a los OGM, pero también hay otros. Estos son el contexto económico, político, social y cultural en el que discursos y argumentaciones serán interpretados, interiorizados. Sin olvidar que en el debate, en los posicionamientos que aparecen, en las afinidades que despiertan o sugieren a'los espectadores de la polémica, subyacen grandes corrientes de fondo, diferentes visiones del mundo, estilos de pensar contrapuestos y consideraciones éticas y morales diferentes. Así, las percepciones públicas sobre la biotecnología aparecen complejas y ambivalentes.

Además, parece que los sentidos de las representaciones sociales nos alejan del terreno en el que se pretenden fijar las opiniones públicas alrededor de los OGM. Las preguntas sobre posicionamiento que las encuestas de opinión hacen a los ciudadanos respecto a este tema enmascaran la complejidad de los contornos en los que se circunscriben y explican esos posicionamientos coyunturales. Estas posiciones surgen de la interiorización que los individuos hacen de los mensajes que reciben, de acuerdo con sus propias pautas interpretativas de la sociedad en la que viven.

Por todo ello, aunque la biotecnología sea una nueva tecnología y la tecnociencia una novedad de nuestro tiempo profundamente transformadora, las perspectivas desde las cuales analizamos sus implicaciones y valoramos sus efectos no tienen nada de nuevo. El debate es viejo, y como siempre, perfectamente actual, porque sigue vigente, porque sigue sin resolverse. Es un debate sobre la naturaleza y el ser humano, sobre el ser humano en la naturaleza, sobre la naturaleza del ser humano. Pero es también un debate sobre las relaciones entre seres humanos, sobre cómo se organizan éstos en sociedad. Y si el conocimiento científico se ha desarrollado de manera espectacular a lo largo del último siglo, y su alianza con la tecnología ha modificado profundamente nuestra tecnosfera, no puede decirse lo mismo de nuestro medio organizativo o social. Einstein observó en su libro Out of my late years, no mucho después de los bombardeos de Hiroshima y Nagasaki, que "nuestra época parece caracterizarse por la perfección de los medios y la confusión de los objetivos". Desde entonces, los medios son cada vez más eficaces y la confusión no parece ser menor.

A lo mejor para que el debate encuentre vías de consenso social entre los agentes y complicidad con la sociedad, sea necesario que se centre de una vez por todas en 
RIS

una cuestión esencial: qué objetivos — cuáles son deseables y cuáles no—y cómo alcanzarlos - con qué medios-. Ésta parece ser la cuestión que atraviesa no sólo el debate entre agentes, sino también, y quizás más importante, la interpretación que del debate ha hecho el resto de la sociedad. Y eso es algo que el debate social sobre los OGM sufre con intensidad, porque es un terreno abonado para retratar contradicciones, desavenencias e inconsistencias de todo tipo.

Mientras ello no suceda, conviene, pues, seguir profundizando en esas pautas que dan sentido a la forma que cobran el debate y las representaciones sociales, y alejarse de las orientaciones interpretativas que produce la dependencia de las herramientas usadas sobre la opinión pública, considerándolas como fuentes de información, pero reconociendo también sus limitaciones y bajando al terreno de las representaciones, verdadero ámbito de interpretación de la naturaleza de las percepciones y actitudes hacia los OGM.

\section{REFERENCIAS BIBLIOGRÁFICAS}

BUSCH, L. (1991), "Biotechnology: Consumer concerns about risks and values", Food Technology, Vol. 46 p.p. $96-101$

HOBAN, T.J. y P. KENDALL (1992), Consumer attitudes about the use of biotechnology in agriculture and food production, United States, Department of Agriculture.

INSTITUTO DE ESTUDIOS SOCIALES AVANZADOS (1991), Biotecnologia y opinión pública en España, Madrid, CSIC.

LÓPEZ CEREZO, J.A. y J.L. LUJÁN (2000), Ciencia y política del riesgo, Madrid, Alianza editorial.

MARRIS, C., B. WYNNE, P. SIMMONS, y S. WELDON (2002), Public Perception of Agricultural Biotechnologies, (PABE), (Informe inédito).

MATEO BOX, J.M. (1993), Biotecnología, agricultura y alimentación, Madrid, OCDE-Ediciones Mundi-Prensa.

MUÑOZ, E. (2000), "Plantas transgénicas y sociedad: unas relaciones complejas", La biotecnología aplicada a la agricultura, Madrid, pp. 247-254

(2001), Biotecnologia y Sociedad: Encuentros y Desencuentros, Madrid, Cambridge University Press.

SWOKA, A.M. (1993), Consumer acceptance of food biotechnology, Hamstra, Research Report, Vol. 137.

ZECHENDORF, B. (1994), “What the public thinks about biotechnology”, Bio/Technology, Vol, 12, pp. 870-875. 